\title{
Progress in deep Markov State Modeling: Coarse graining and experimental data restraints
}

\author{
Andreas Mardt ${ }^{1}$ and Frank Noé ${ }^{1,2,3}$ \\ ${ }^{1)}$ Department of Mathematics and Computer Science, Freie Universität Berlin, Berlin, \\ Germany \\ ${ }^{2)}$ Department of Physics, Freie Universität Berlin, Berlin, Germany \\ ${ }^{3)}$ Department of Chemistry, Rice University, Houston TX, 77005, United State ${ }^{\text {a) }}$
}

Recent advances in deep learning frameworks have established valuable tools for analyzing the long-timescale behavior of complex systems such as proteins. Especially the inclusion of physical constraints, e.g. timereversibility, was a crucial step to make the methods applicable to biophysical systems. Furthermore, we advance the method by incorporating experimental observables into the model estimation showing that biases in simulation data can be compensated for. We further develop a new neural network layer in order to build an hierarchical model allowing for different level of details to be studied. Finally, we propose an attention mechanism which highlights important residues for the classification into different states. We demonstrate the new methodology on an ultralong molecular dynamics simulation of the Villin headpiece miniprotein.

\section{INTRODUCTION}

The thermodynamics and kinetics of large biological macromolecules can be studied in full spatiotemporal detail with molecular dynamics (MD) simulations 1 [1] The combined progress in high-throughput MD simulations and in analysis frameworks such as Markov state models $\left(\mathrm{MSMs}^{10} \sqrt{21}\right.$, Master-equation models $\sqrt{224}$ and closely related approaches $\frac{1725}{33}$ has led to the successful characterizations of the kinetics of folding proteins $14|34| 35$, protein-ligand association $\frac{36}{39}$, and even protein-protein association 40 . These methods benefit from the facts that they do not require a rigorous definition of reaction coordinates 41 and allow the extraction of experimental observables which can be connected to structural changes of the system 42 , 48 .

Additionally, the MSM approach can handle nonequilibrium data by approximating the transition density $p_{\tau}(\mathbf{x}, \mathbf{y})$ of a Markov process:

$$
p_{\tau}(\mathbf{x}, \mathbf{y})=\mathbb{P}\left(\mathbf{x}_{t+\tau}=\mathbf{y} \mid \mathbf{x}_{t}=\mathbf{x}\right),
$$

that models the conditional probability of the system transitioning to configuration $\mathbf{y}$ when starting in configuration $\mathbf{x}$ after a time interval $\tau$, called lag-time.

Whereas the construction of MSMs has previously been done manually via a challenging and potentially error prone pipeline of feature selection, dimension reduction, clustering, estimating a transition matrix $\mathbf{P}$, etc, it can be largely automated using variational approaches that optimize MSMs to best resolve the rare event processes 49150 . Firstly, the variational approach for conformation dynamics (VAC) has been developed to find optimal model parameters5152. It was generalized by the variational approach for Markov processes (VAMP) which increases the scope to non-reversible and non-stationary dynamics 53 .

\footnotetext{
a) correspondence to frank.noe@fu-berlin.de
}

An end-to-end deep learning method called VAMPnets was proposed which replaces the MSM building through training a neural network mapping the configurations $\mathbf{x}$ to a low dimensional state space $\boldsymbol{\chi}(\mathbf{x}))^{54}$. This state space resembles the state space of an ordinary MSM by having a softmax output function which results in fuzzy state assignments of each configuration, which can be interpreted as state probabilities $\mathbf{p}(t)=\boldsymbol{\chi}\left(\mathbf{x}_{t}\right)$. A VAMPnet can be trained by maximizing the VAMP score to find an optimal state space allowing the linear propagation of the state probabilities via a transition matrix:

$$
\mathbf{p}^{T}(t+\tau)=\mathbf{p}^{T}(t) \mathbf{P}
$$

which gives access to the kinetics of the system by studying the properties of $\mathbf{P}$. However, the matrix is not guaranteed to satisfy stochastic properties, i.e. it can have negative or entries larger than 1 . Further advances have been proposed, which do not aim to replace the whole pipeline, but instead finding optimal features to build an ordinary MSM via the rest of the pipeline $e^{55}$, or which propose transferable feature functions across chemical space ${ }^{56}$. Recently, a method was introduced which allows VAMPnets with physical constraints to be constructed, addressing two major issues:

1. The transition matrix $\mathbf{P}$ can be enforced to have exclusively non-negative entries thus being a stochastic matrix. Therefore, further analysis using transition path theory is possible ${ }^{57}[59$.

2. Time-reversibility (detailed balance) can be enforced in the model when the underlying dynamics obeys microscopic reversibility, but the data is sampled out of equilibrium.

The method allows to select either of the two constraints independently, which enables the user to build four different classes of models. In this work we will focus on the reversible deep MSM (revDMSM), which has been already successfully employed to a disordered protein 60 , to applying both constraints and thereby allowing the 
application of transition path theory to study rates of interesting processes.

However, in the field of MSMs for protein analysis further developments have been made which need to be transfered to the case of a deep learning framework. Here, we address two major limitations of VAMPnets and deep MSMs that have been addressed for classical MSMs and are important ingredients in MSM applications.

On the one hand, several methods have been proposed to coarse-grain an existing MSM using spectral clustering $\frac{6163}{61}$, which allows the user to study the system on different degrees of detail. Here, we develop a new layer structure for neural networks to coarse-grain the existing model by still obeying the constraints and keeping it trainable end-to-end.

On the other hand, it has been proposed and showed that incorporating existing experimental information into the model estimation helps to overcome systematic errors in the force field $15|64| 70$. Consequently, we show how different classes of observables can be included into the training routine of a revDMSM.

Furthermore, the black box nature of deep learning frameworks fueled the development of methods aiming at understanding individual decisions of the neural network ${ }^{71}$ 80 . These fall into two categories, the post hoc analysis of the network and the simultaneously trained attention mechanism. Here, we focus on the later to optimize it for the application on proteins in the context of a revDMSM.

In summary, we will advance the revDMSM by the following contributions based on a study of a small protein:

1. Comparison between building a MSM via a revDMSM and a VAMPnet.

2. Analyzing the slow processes.

3. Applying transition path theory to estimate folding and unfolding rates.

4. Deriving an algorithm in order to built an hierarchical model to allow an easier interpretation of the resulting model.

5. Proposing an attention mechanism which supports the understanding of how important each residue is for the dynamic classification.

6. Developing an algorithm to incorporate experimental observations into the model estimation.

\section{METHODS}

As proposed in Ref. 81 the transition density of a Markov process is approximated by:

$$
p_{\tau}(\mathbf{x}, \mathbf{y})=\chi(\mathbf{x})^{T} \mathbf{S} \chi(\mathbf{y}) \chi(\mathbf{y})^{T} \mathbf{u} \rho_{1}(\mathbf{y}),
$$

which models the probability of the system transitioning to configuration $\mathbf{y}$ when starting in configuration $\mathbf{x}$

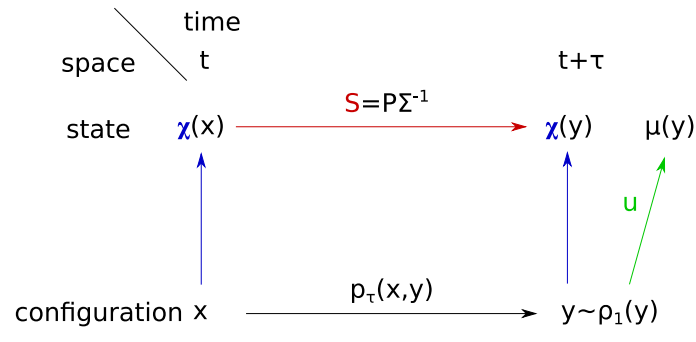

Figure 1. Schematic of the probability model given by a revDMSM. The conditional probability distribution $p_{\tau}(\mathbf{x}, \mathbf{y})$ of jumping to configuration $\mathbf{y}$ after a time interval $\tau$ given a system in configuration $\mathbf{x}$ is approximated by transferring the estimation into a trainable state space $\chi(\mathbf{x})$. The starting configuration $\mathbf{x}$ is mapped to the state space $\chi(\mathbf{x})$ and consecutively propagated by the transition matrix $\mathbf{P}$. The probability of the propagated state vector belonging to a configuration $\mathbf{y}$ is given by the scalar product with the state vector $\chi(\mathbf{y})$ weighted by the stationary distribution $\mu(\mathbf{y})$. The stationary distribution is given via the trainable vector $\mathbf{u}$ and the transition matrix via the trainable matrix $\mathbf{S}=\mathbf{P} \boldsymbol{\Sigma}^{-1}$, where $\boldsymbol{\Sigma}=\int \boldsymbol{\chi}(\mathbf{y}) \mu(\mathbf{y}) \boldsymbol{\chi}(\mathbf{y})^{T} \mathrm{~d} \mathbf{y}$ normalizes the probability distribution, i.e. integrating the distribution over all possible configurations $\mathbf{y}$ evaluates to 1 .

after a time interval $\tau$, called lag-time. The function $\chi(\mathbf{x})$ represented by a neural network maps a configuration to a fuzzy state space of dimension $m$ similar to a VAMPnet ${ }^{54}$. The trainable vector $\mathbf{u} \in \mathbb{R}^{m}$ reweights the empirical distribution $\rho_{1}(\mathbf{y})$ to the learned stationary distribution:

$$
\mu(\mathbf{y})=\chi(\mathbf{y})^{T} \mathbf{u} \rho_{1}(\mathbf{y}) .
$$

The trainable matrix $\mathbf{S} \in \mathbb{R}^{m \times m}$ gives access to the transition matrix $\mathbf{P}$ between the states:

$$
\mathbf{P}=\mathbf{S} \boldsymbol{\Sigma}
$$

$$
\begin{aligned}
\boldsymbol{\Sigma} & =\int \chi(\mathbf{y}) \mu(\mathbf{y}) \chi(\mathbf{y})^{T} \mathrm{~d} \mathbf{y} \\
& =\int \chi(\mathbf{y}) \rho_{1}(\mathbf{y}) \chi(\mathbf{y})^{T} \mathbf{u} \chi(\mathbf{y})^{T} \mathrm{~d} \mathbf{y}
\end{aligned}
$$

being the equilibrium covariance matrix of $\chi(\mathbf{y})$ (Fig. 1).

\section{A. Reversible deep Markov State Models}

Since $\chi(\mathbf{x})$ should be a fuzzy state assignment and the stationary distribution and the transition matrix have to be normalized, the following constraints have to be fulfilled 81 :

1. Normalized state vector: $\boldsymbol{\chi}(\mathbf{x})^{T} \mathbf{1}=1$.

2. Normalized stationary distribution: $\bar{\chi}^{T} \mathbf{u}=1$, where $\bar{\chi}=\mathbb{E}\left[\chi\left(\mathbf{x}_{t+\tau}\right)\right]$ is the empirical state probability, which results in $\int \mu(\mathbf{y}) \mathrm{d} \mathbf{y}=\bar{\chi}^{T} \mathbf{u}=1$. 
3. Normalized transition matrix: $\mathbf{S C}_{\tau \tau}^{\prime} \mathbf{u}=\mathbf{1}$ where $\mathbf{C}_{\tau \tau}^{\prime}=\mathbb{E}\left[\boldsymbol{\chi}\left(\mathbf{x}_{t+\tau}\right) \boldsymbol{\chi}\left(\mathbf{x}_{t+\tau}\right)^{\top}\right]$ is the empirical covariance matrix of $\chi\left(\mathbf{x}_{t+\tau}\right)$. As a result the transition matrix preserves probability mass by means of $\mathbf{P} \mathbf{1}=\mathbf{S C}_{\tau \tau}^{\prime} \mathbf{u}=\mathbf{1}$.

The general approach allows the training of different classes of models. Here, we will focus on the revDMSM, where the matrix $\mathbf{S}$ has to be additionally symmetric $\mathbf{S}=\mathbf{S}^{T}$ and $\mathbf{S}, \mathbf{u}$, and $\chi$ have to be non-negative. The constraints will be matched by a proper choice of architectures for the different trainable parts.

Two different losses for training are introduced: the maximum likelihood (ML), where the probability of observing the data according to the model is maximized, and the VAMP-E score, which is used throughout the paper. The training procedure of such a model includes the pretraining of a VAMPnet, which we have slightly modified (Appendix VIID).

It has been proven that the proposed model is an universal approximator for reversible Markov processes ${ }^{81}$, which makes it therefore a promising candidate to study biological systems. Furthermore, it was demonstrated that it yields asymptotically unbiased results even in the case of many short trajectories allowing the analysis of parallel simulated data.

\section{B. Incorporating experimental observables in the model estimation}

For an experiment such as fluorescence, chemical shift in NMR, IR spectroscopy the conformational dynamics are mapped onto an observable $a$. In the following we assume that $a$ has a scalar value associated with conformation $\mathbf{x}, a(\mathbf{x})$. The generalization to vector- or tensorvalued observables is straightforward. In equilibrium the ensemble average will be measured by the experiment, which can be approximated by a weighted sum with the equilibrium weights $\mu\left(\mathbf{x}_{t}\right)$ for simulation data with $\sum_{t=1}^{T} \mu\left(\mathbf{x}_{t}\right)=1$ :

$$
\mathbb{E}[a] \approx \sum_{t=1}^{T} \mu\left(\mathbf{x}_{t}\right) a\left(\mathbf{x}_{t}\right) .
$$

Since simulations carry a systematic bias due to the force field approximation and finite sampling, the estimated value from a simulation may differ from the experiment. However, if experimental information for some observables is available, they can be incorporated into the model estimation. By possibly removing the bias from the simulation calculations of observables not included in the estimation procedure could be improved. Therefore, we propose to extent the loss function by the objective to match the observables measured by experiments:

$$
L_{\text {total }}=L_{\mathrm{MSM}}+\sum_{i} \lambda_{i}\left\|O_{i}-\mathbb{E}\left[a_{i}\right]\right\|^{2},
$$

where $O_{i}$ is the ensemble average measured in experiment for the $i$ th observable, where each observable can be weighted by $\lambda_{i}$ which encodes uncertainty about the observation. $L_{\mathrm{MSM}}$ is either the VAMP-E or ML loss as proposed in the original paper.

Depending on where the bias is to be expected, the loss can be used to train for all instances $\chi, \mathbf{u}, \mathbf{S}$. Otherwise, if e.g. the state definition seems trustworthy and a reweighting should be enough to counteract the bias $\mathbf{u}$ could be trained by the additional loss keeping $\chi$ and $\mathbf{S}$ fixed.

The same approach can be used for available kinetic information through time-correlation experiments. The expectation value of these time-correlations can be expressed via the simulation and the model as:

$$
\begin{aligned}
\mathbb{E}[a(t) a(t+k \tau)] & \approx \sum_{t_{1}=1}^{T} \mu\left(\mathbf{x}_{t_{1}}\right) a\left(\mathbf{x}_{t_{1}}\right) \sum_{t_{2}=1}^{T} p_{k \tau}\left(\mathbf{x}_{t_{1}}, \mathbf{x}_{t_{2}}\right) a\left(\mathbf{x}_{t_{2}}\right) \\
& =\mathbf{a}^{T} \mathbf{X}^{k}(\tau) \mathbf{a}
\end{aligned}
$$

where a gives the average value of the observable within each state and $\mathbf{X}^{k}(\tau)$ is the unconditional probability to jump between the states $\frac{15}{15}$. We can estimate both quantities via our model as:

$$
\begin{aligned}
\mathbf{a}_{i} & =\sum_{t} a\left(\mathbf{x}_{t}\right) \frac{\chi_{i}\left(\mathbf{x}_{t}\right) \mu\left(\mathbf{x}_{t}\right)}{\sum_{t^{\prime}} \chi_{i}\left(\mathbf{x}_{t^{\prime}}\right) \mu\left(\mathbf{x}_{t^{\prime}}\right)}, \\
\mathbf{X}(\tau) & =\boldsymbol{\Sigma}_{t} \mathbf{P},
\end{aligned}
$$

with $\boldsymbol{\Sigma}_{t}$ being the equilibrium covariance matrix of $\chi\left(\mathbf{x}_{t}\right): \boldsymbol{\Sigma}_{t}=\int \boldsymbol{\chi}\left(\mathbf{x}_{t}\right) \mu\left(\mathbf{x}_{t}\right) \boldsymbol{\chi}\left(\mathbf{x}_{t}\right)^{T} \mathrm{~d} \mathbf{x}_{t}$. In the case of a normalized time correlation with $\bar{a}=a-\mathbb{E}[a]$ the term reads:

$$
\frac{\mathbb{E}[\bar{a}(t) \bar{a}(t+\tau)]}{\mathbb{E}\left[\bar{a}(t)^{2}\right]}=\frac{\overline{\mathbf{a}}^{T} \mathbf{X}^{k}(\tau) \overline{\mathbf{a}}}{\sum_{t=1}^{T} \mu\left(\mathbf{x}_{t}\right) \bar{a}^{2}\left(\mathbf{x}_{t}\right)} .
$$

Additionally, it is of great interest to incorporate experimentally measurable relaxation timescales $t_{i}$ into the model which are directly related to the eigenvalues $\lambda_{i}$ of the transition matrix $\mathbf{P}$ via $t_{i}=-\log \left(\lambda_{i}\right) / \tau$. However, the transition matrix $\mathbf{P}$ is not Hermitian and therefore estimating gradients of its eigenvalues is numerically unstable and not supported by the main deep learning tools such as PyTorch or tensorflow. Fortunately, from Eq. (5) it follows:

$$
\overline{\mathbf{S}}=\boldsymbol{\Sigma}^{1 / 2} \mathbf{S} \boldsymbol{\Sigma}^{1 / 2}=\boldsymbol{\Sigma}^{1 / 2} \mathbf{P} \boldsymbol{\Sigma}^{-1 / 2},
$$

which shows that $\mathbf{P}$ is similar to the Hermitian matrix $\overline{\mathbf{S}}$ and therefore we can optimize for the eigenvalues of $\overline{\mathbf{S}}$ instead. A connection between folding/unfolding rates and timescales is given in Appendix VIIC.

\section{Coarse graining}

For interpreting and understanding the model it can be helpful to build an hierarchical state splitting of the 
model, where the system can first be studied on a coarse and subsequently on a finer level. This structure can be obtained by training independent models with different output sizes and comparing the resulting states 54 . We propose instead to make use of the given loss and learn the coarse graining on the fly. Given a model with $m$ output nodes we want to learn a matrix $\mathbf{M} \in \mathbb{R}^{m x n}$, which maps the output of the model $\chi^{m} \in \mathbb{R}^{m}$ to a coarser model with $n<m$ states via:

$$
\left(\chi^{n}\right)^{T}=\left(\chi^{m}\right)^{T} \mathbf{M}
$$

The coarse graining matrix can be trained by using the proposed loss functions or simply the VAMP-2 score in the case of a VAMPnet. Furthermore, we want that $\mathbf{M}_{i j}>0$ and $\sum_{j} \mathbf{M}_{i j}=1$, which is ensured by defining trainable weights $\mathbf{m} \in \mathbb{R}^{m \times n}$ and squeeze them through a softmax function:

$$
\mathbf{M}_{i j}=\frac{\exp \left(m_{i j}\right)}{\sum_{l} \exp \left(m_{i l}\right)} .
$$

However, when training a reversible model with the parameters $\mathbf{u}^{m}$ and $\mathbf{S}^{m}$ there is no need to retrain $\mathbf{u}^{n}$ and $\mathbf{S}^{n}$ for the finer model. Instead, it is preferable to ensure consistency between the models regarding the stationary and transition density. Starting with the stationary distribution $\mu^{m}(\mathbf{x}) \stackrel{!}{=} \mu^{n}(\mathbf{x})$, it follows:

$$
\begin{aligned}
\left(\chi^{m}(\mathbf{x})\right)^{T} \mathbf{u}^{m} & =\left(\boldsymbol{\chi}^{n}(\mathbf{x})\right)^{T} \mathbf{u}^{n}=\left(\boldsymbol{\chi}^{m}(\mathbf{x})\right)^{T} \mathbf{M} \mathbf{u}^{n} \\
\Rightarrow \mathbf{u}^{m} & =\mathbf{M} \mathbf{u}^{n}
\end{aligned}
$$

which can be solved via the pseudoinverse of $\mathbf{M}=$ $\mathbf{U D V}^{T}$ :

$$
\mathbf{u}^{n}=\mathbf{V} \mathbf{D}^{* T} \mathbf{U}^{T} \mathbf{u}^{m}=\mathbf{G} \mathbf{u}^{m}
$$

where $\mathbf{D}^{*}$ has the reciprocal value of every non-zero element in $\mathbf{D}$.

A similar derivation can be done for $\mathbf{S}$ given that $p_{\tau}^{m}(\mathbf{x}, \mathbf{y}) \stackrel{!}{=} p_{\tau}^{n}(\mathbf{x}, \mathbf{y})$ and $\mu^{m}(\mathbf{x})=\mu^{n}(\mathbf{x})$ :

$$
\begin{aligned}
\left(\chi^{m}(\mathbf{x})\right)^{T} \mathbf{S}^{m} \chi^{m}(\mathbf{y}) & =\left(\chi^{n}(\mathbf{x})\right)^{T} \mathbf{S}^{n} \chi^{n}(\mathbf{y}) \\
& =\left(\chi^{m}(\mathbf{x})\right)^{T} \mathbf{M S}^{n} \mathbf{M}^{T} \chi^{m}(\mathbf{y}) \\
\Rightarrow \mathbf{S}^{m} & =\mathbf{M} \mathbf{S}^{n} \mathbf{M}^{T} \\
\mathbf{S}^{n} & =\mathbf{G} \mathbf{S}^{m} \mathbf{G}^{T}
\end{aligned}
$$

However, both $\mathbf{u}^{n}$ and $\mathbf{S}^{n}$ still need to fulfill the constraints. Since the pseudoinverse represents the least square solution, the constraints could be violated. Therefore, we propose to renormalize the previous results 81 .

$$
\begin{aligned}
\mathbf{u}^{n} & =\frac{\mathbf{u}^{n}}{\left(\bar{\chi}^{n}\right)^{T} \mathbf{u}^{n}} \\
\mathbf{S}^{n} & =\mathbf{S}^{n}+\operatorname{diag}(\mathbf{s}) \\
s_{i} & =\frac{1-\sum_{k} S_{i k}^{n} v_{k}^{n}}{v_{i}^{n}} .
\end{aligned}
$$

In order to make the result more robust it is favorable to train $\mathbf{u}^{m}, \mathbf{S}^{m}, \mathbf{M}_{i}$ simultaneously for the sum of the VAMP-E scores of all models. However, if it is preferable to retrain $\mathbf{u}^{n}$ and $\mathbf{S}^{n}$, the consistency expressions should be taken into account in the loss function.

Furthermore, we suggest to initiate the weights of $\mathbf{M}$ according to the $\mathrm{PCCA}+$ memberships ${ }^{82}$. Thereby, we avoid the network becoming stuck in an unfavorable solution during the beginning of training.

\section{RESULTS}

\section{A. Overview}

Below we demonstrate how a revDMSM can be applied and expanded by our proposed methods to the Villin dataset provided by ${ }^{35}$ (folded structure Fig. 2 a). The input for $\boldsymbol{\chi}$ is $\exp (-\mathbf{d})$, where $\mathbf{d}$ is the minimal heavy atom distance between all residues 54 . Firstly, we will study the ability of the method to guarantee a reversible MSM and compare the result against a VAMPnet in a low data regime. Afterwards, we will build an hierarchical model and applying our proposed attention mechanism to it. Based on this, we study folding and unfolding rates via transition path theory. Finally, we will look at the effects of including ground truth observables into the training.

\section{B. Implementation}

The methods were implemented using PyTorch 83 . For the full code and details about the neural network architecture, hyperparameters and training routine, please refer to https://github.com/markovmodel/deepmsm. In

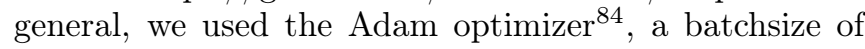
10000 , and a 6 layer deep neural network with a constant width of 100 nodes with the ELU activation function for $\chi$. When using an attention network it has the same architecture as $\chi$ except of the output size and a window size of 4 motivated by the fact that the shortest beta strands and a helix turn is about four residues long.

The whole simulation data is randomly split threefold into $70 \%$ training, $20 \%$ validation and $10 \%$ test set data. The validation data is used to tune hyperparameters and enact an early stopping mechanism. The results are reported for the test set.

\section{Obtaining real eigenvalues and positive entries in the transition matrix via a revDMSM}

To simulate an insufficient sampled example, we used a skip of 100 frames (1frame= $20 \mathrm{~ns}$ ) and 10 output nodes. We train a regular VAMPnet and a revDMSM on the training data with an early stopping given by the performance on the validation set and estimate the resulting transition matrix on the test set at a time-lag of 
a)

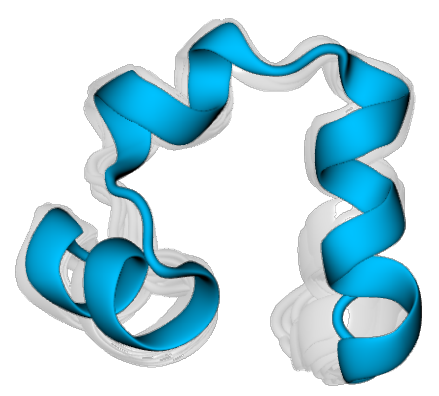

b)

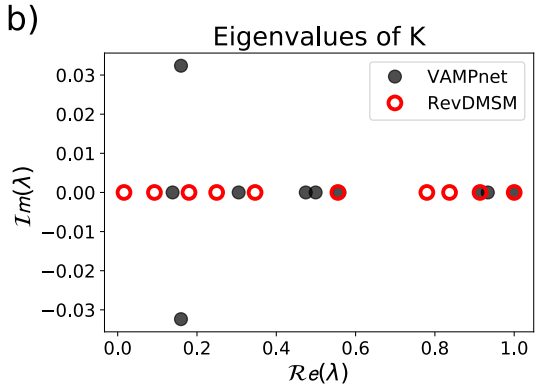

c)

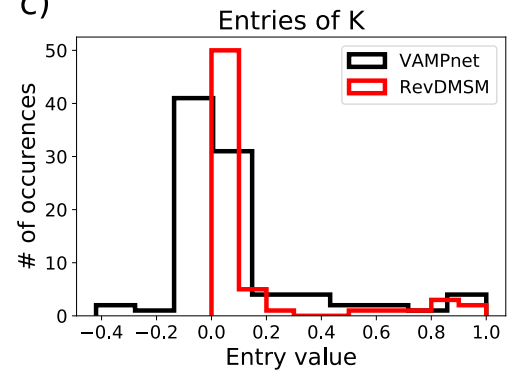

Figure 2. Incorporating constraints on the matrix $\mathbf{P}$ to enforce a reversible model in the regime of poorly sampled systems. a) Folded structure of Villin b) Imaginary and real part of the spectrum and c) entries of the matrix $\mathbf{P}$ of the non-reversible VAMPnet and the revDMSM with a skip of 100 frames and 10 states ( 1 frame $=\tau=20 \mathrm{~ns}$ ).

$\tau=20 \mathrm{~ns}$. The eigenvalues of the transition matrix are always real for the revDMSM, whereas in the case of a VAMPnet pairwise complex eigenvalues may occur (2 b). Furthermore, the distribution of the entries demonstrate how the revDMSM in contrast to the VAMPnet guarantees values between 0 and 1 , which can be interpreted as probabilities $(2 \mathrm{c})$. The insufficient sampling leads in the case of the VAMPnet to a non-reversible model, where the transition matrix is not a stochastic matrix. The revDMSM model does not suffer from these shortcomings, nevertheless the constraints imposed on the model result in slightly lower eigenvalues, which can be expected since the constraints impair the ability to approximate the eigenfunctions of the underlying operator.

\section{Building a MSM and testing its validity for a VAMPnet and a revDMSM}

Based on the same data as above but with a skip of 25 frames ( 1 frame $=\tau=5 \mathrm{~ns}$ ), we built a MSM with 4 output nodes using a revDMSM and a VAMPnet, respectively. By inspecting the state network connected by their transition probabilities we observe again negative transition probabilities for the VAMPnet (red arrows Fig. 3 b). However, the implied timescale and the CK test confirm the ability of both models to predict the long-time kinetics. Furthermore, the models agree upon the timescales within the 70th percentile estimated over 10 runs and discover similar metastable states, where 10 aligned representative structures are depicted next to each state (Fig. 3 a, b). Although each model uses different trained feature functions $\chi$ they both identify a folded state (F), an unfolded state (U), a partially folded state $(\mathrm{PF})$, and a misfolded state $(\mathrm{M})$ characterized by a helix including the amino acids 20LEU and 21PRO which form a coil in the folded state.

\section{E. Building an hierarchical model with an interpretable attention mechanism}

In order to demonstrate the application of an hierarchical model we coarse grain a 4 state revDMSM to a 3 state and consecutively to a 2 state model. Additionally, we incorporate an attention mechanism into the architecture (Sec. VIIA). After training the 4 state model at $\tau=50$ ns we train the coarse-graining matrices with the VAMP-E score consecutively with the pseudoinverse method. Finally, we simultaneously update $\mathbf{u}$ and $\mathbf{S}$ from the 4 state model and the two coarse graining matrices to maximize the sum of the VAMP-E scores of all three models.

For the estimation of the implied timescales of all models it is sufficient to exclusively retrain $\mathbf{u}$ and $\mathbf{S}$ from the 4 state model to optimize the sum of the individual scores. The 3 and 2 state model conserve both the highest timescales as expected (Fig. 4 top row). Thereby, the 2 state model, where the unfolded state is missing, indicates that the slowest timescale is not connected to the folding process. Instead, the state of the unfolded structure appears in the 3 state model emphasizing the nature of folding of the second timescale.

Below the timescales we depict a graphical representation of the hierarchical model, where each node represents a state, where the same nomenclature is used as above. The connections between the nodes represent the coarse graining matrix, where the numbers encode the probability that the state belongs to the coarse grained state (connections with a probability less than $1 \%$ are not displayed). For reasons of clarity we added additionally visual representations of the matrices beside it, where the color encodes the probability (white-low, red-high). There, it becomes evident that the coarse-grain matrices have a very sparse structure. The large values seem to be in agreement with the observation in the original paper that revDMSMs tend to rather hard assignments of states. However, the hard assignment supports the interpretation of an hierarchical model.

Next to the nodes we added the structures of Villin with the highest probability for that particular state. 
a)
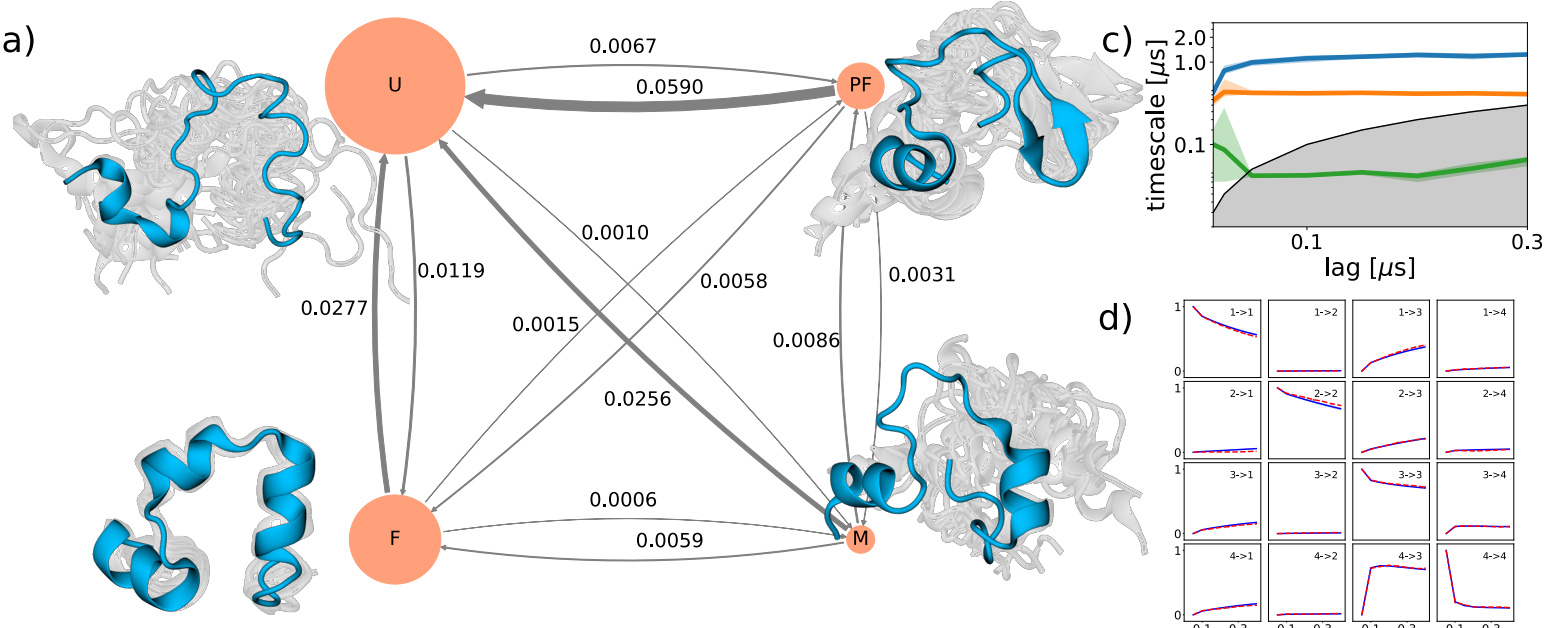

d)
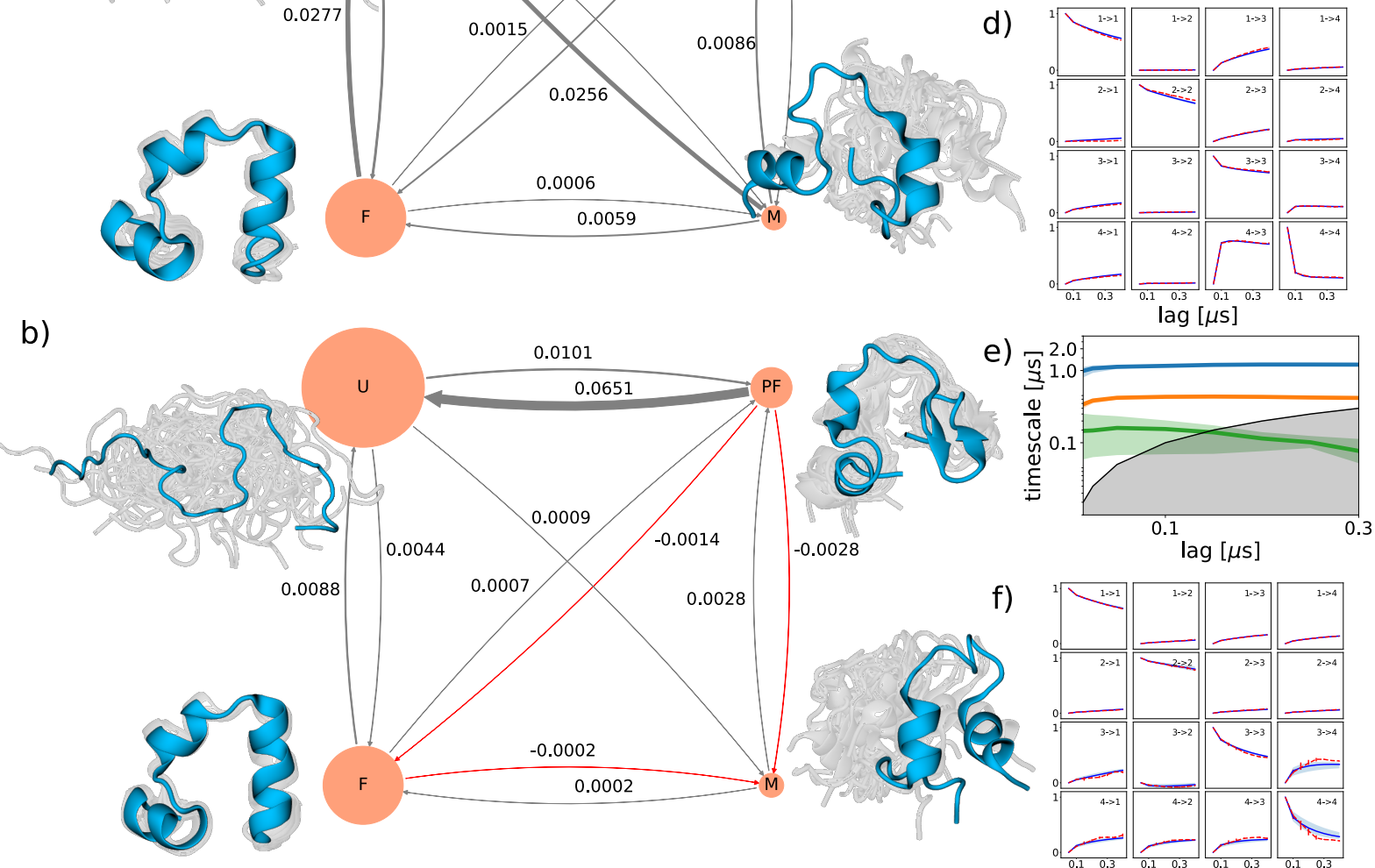

f)

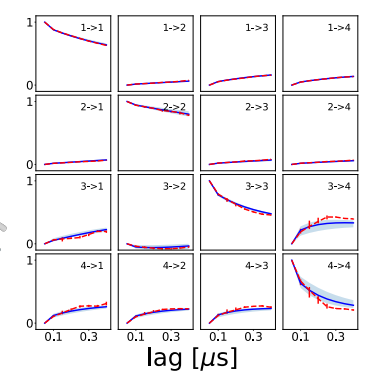

Figure 3. Comparison of building a MSM with non-reversible VAMPnets and revDMSM with a skip of 25 frames $(1$ frame $=$ $5 \mathrm{~ns}$ ) and 4 states. State network of the revDMSM model a) and VAMPnet b), the size of a state corresponds to the stationary distribution, the arrows are the transition rates of the $\mathbf{P}$ matrix (red arrows indicate negative entries). Additionally, 10 representative structures aligned according to their secondary structure are shown next to the states. Although trained independently both feature functions identify a folded state $(\mathrm{F})$, and unfolded state (U), a partially folded state (PF), and a misfolded state (M). Model validation through the implied timescales of the c) revDMSM and e) VAMPnets model and the CK-test d) and f) at a base model estimated at $\tau_{\mathrm{MSM}}=50 \mathrm{~ns}$. Errors are estimated over 10 runs.

There, the hierarchical splitting is visible: structure elements of the higher model hierarchy are preserved in the lower level.

Furthermore, we depict the attention weight of each residue in the color scheme of the structures (red-high, white-low). It is worth mentioning, that the attention mechanism needs to highlight areas which are important to distinguish all four metastable states. This implies that the absence of a specific secondary structure could be important and therefore highlighted.

Remarkably, our attention mechanism detects 13ARG as important for the folded structure (F), which we found aligns well with the folding process (Sec. IIIH). The misfolded state $(\mathrm{M})$ shows high attention at the amino acids 20LEU and 21PRO being part of a helix which form a coil in the folded state. The last residues of the chain seem to be bad descriptors for the dynamics which seems reasonable due to their more flexible nature.

In general, the network assigns high attention mainly to regions where states have themselves secondary structure or where they lack the structure other states have, e.g. the middle helix of the folded state.

\section{F. Approximation of the leading eigenfunctions via a revDMSM}

Driven by the observation that the slowest process might not be the folding process, we test the ability of the above revDMSM to approximate the two slowest eigenfunctions of the 4 state model of Villin. In order to visualize the process the frames are ordered by their 

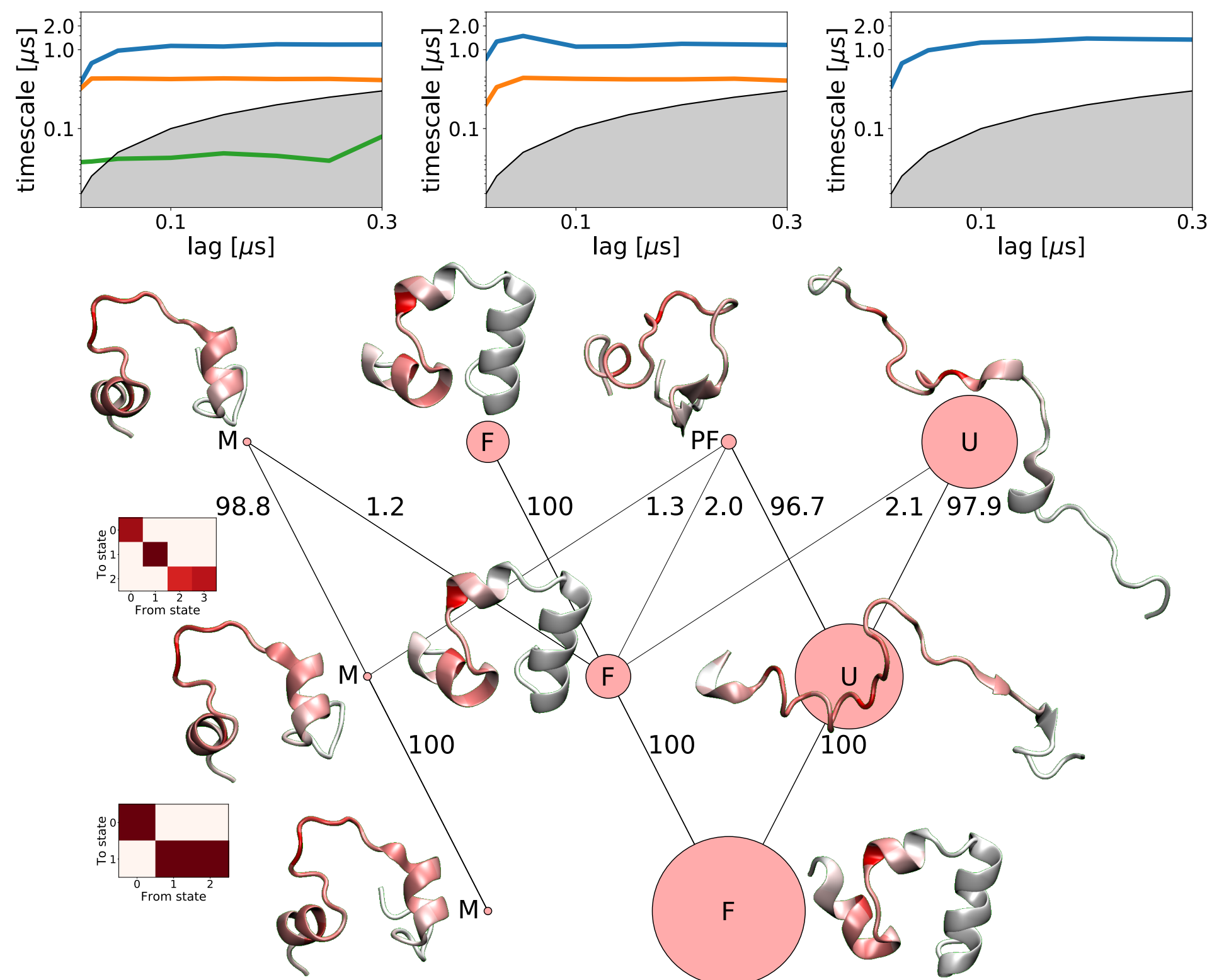

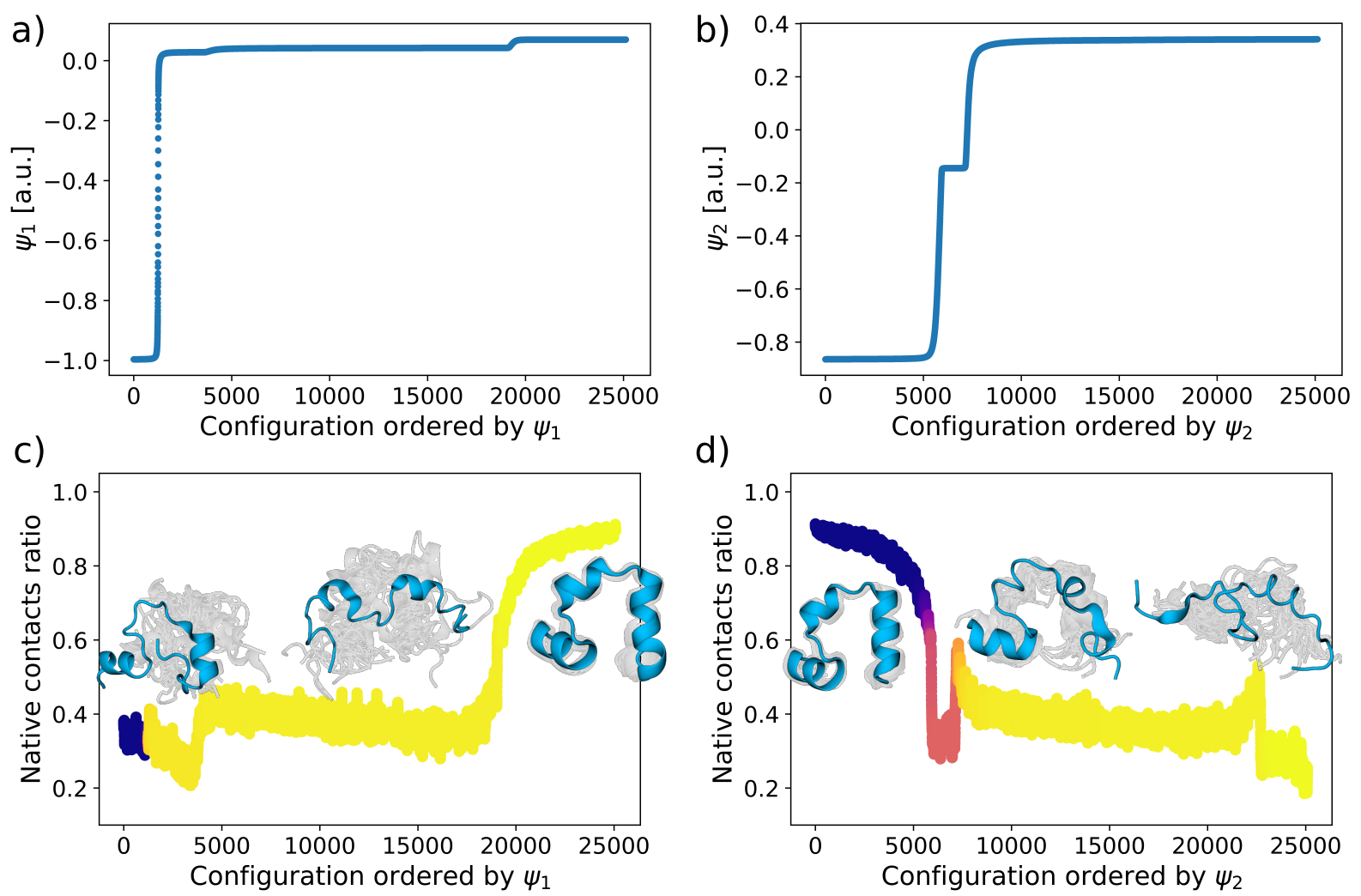

d)

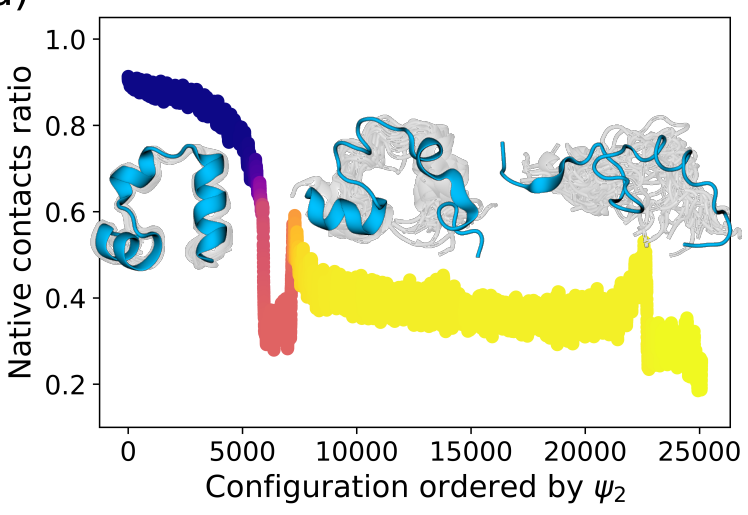

Figure 5. Estimating the two slowest eigenfunctions with a revDMSM on the Villin dataset with a skip of 25 frames $(\tau=50$ ns $)$ and 4 states. a), b) Eigenfunctions against frames ordered by their eigenfunction value (fake trajectory along the process). c), d) The ratio of formed native contacts with a mean window over 30 frames is plotted against the same $\mathrm{x}$-axis, where the color encodes the value of the eigenfunction. Representative structures for three regions are shown.

\section{G. Estimation of folding rates}

We can study via transition-path-theory (TPT) the folding and unfolding rates of the system. We built a three state revDMSM, where $\chi$ was pretrained at a time-lag of $\tau=5 \mathrm{~ns}$ and $\mathbf{u}$ and $\mathbf{S}$ at a lag-time of $\tau=100 \mathrm{~ns}$. The model has to be constituted of at least three states because the folding process is the second slowest timescale in the Villin trajectory. Given the model we estimate from the transition matrix $\mathbf{P}$ the mean first passage time with the PyEMMA package ${ }^{86}$ for the folding and unfolding process:

$$
\begin{aligned}
\tau_{\text {folding }} & =(3.0 \pm 0.3) \mu \mathrm{s}, \\
\tau_{\text {unfolding }} & =(1.0 \pm 0.1) \mu \mathrm{s},
\end{aligned}
$$

where the error is given via the standard deviations over 10 runs. The original paper reports a folding rate of $(2.8 \pm 0.5) \mu \mathrm{s}$ and unfolding rate of $(0.9 \pm 0.2) \mu \mathrm{s}$ which is in perfect agreement with our findings, although they utilize a handcrafted definition of states and simply measure the average lifetime of the folded and unfolded state and the transition time as the average of all events in the trajectory 35 .

\section{H. Estimating deep MSMs with experimental restraints}

In order to mimic the situation of having ground truth values preferably from an experiment but biased simulation data for model estimation, we treat expectation values from the whole long simulation as ground truth. However, we imitate the situation of a simulation which overestimates transition energy barriers compared to the ground truth by removing three fourth of folding and unfolding events from the full trajectory. We detect these events by inspecting the sign changes of the second slowest eigenfunction.

Since we perturbed by our data modification the folding/unfolding process, we focus on observables related to it. Some of them serve as additional information for training, the others as a validation set to compare the performance on them:

1. The kinetics via the implied timescales (ITS) of the two slowest processes.

2. The stationary distribution of three predefined states.

3. The folding and unfolding rates via TPT analysis. 

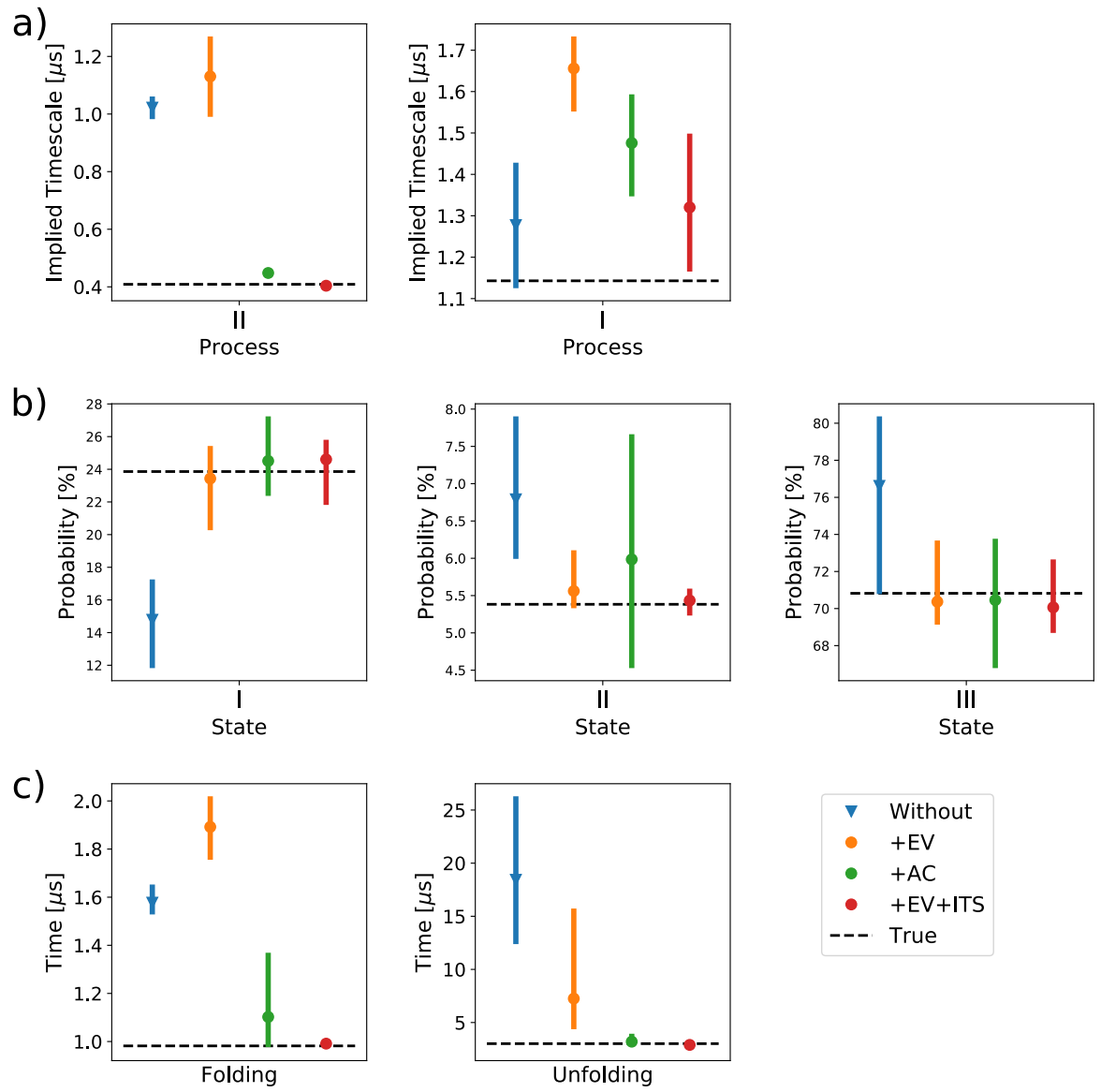

Figure 6. Estimating observables with biased data and comparing estimates between models incorporating some of them into the training routine (orange, green, red) and a model incorporating none (blue). The data is biased by removing folding/unfolding events from the trajectory to simulate a probable higher transition barrier originating from possible erroneous force fields. The true values (dashed black line), ideally from an experiment, were estimated from a model trained on the whole unbiased data set. a) two slowest timescales, where process II is the interesting folding process, which was used for the red model as training observable. Therefore, the better approximation of that process is expected. b) state probabilities and therefore a stationary observable, which was not used during training. However, using the expectation value of the contact 1LEU-13ARG being formed improves the estimation of the stationary properties (orange, red). c) folding and unfolding rates estimated via the transition path theory not used for training, where incorporating the timescale or the autocorrelations of the contact 1LEU-13ARG staying formed or unformed improves the estimation significantly (red and green). The error bars indicate the 68 th percentile.

4. The expectation value (EV) of contact 1LEU$13 \mathrm{ARG}$ being formed.

5. The autocorrelation (AC) of the contact 1LEU13ARG staying formed or unformed.

The contact 1LEU-13ARG is chosen because it correlates well with the folding eigenfunction and could be possibly experimentally observed by attaching fluorescence labels and conducting an fluorescence correlation experiment. A contact is said to be formed if the minimal residue distance is shorter than $0.45 \mathrm{~nm}$ :

$$
a_{t}= \begin{cases}1 & \text { if } d(t)_{1-13}<0.45 \mathrm{~nm} \\ 0 & \text { otherwise }\end{cases}
$$

The three predefined states origin from the classification of the model from Sec. IIIG. The observation of the contact not being formed is simply $b_{t}=1-a_{t}$.

Both models, with (further called observable model) or without (ordinary model) additional observable, take the same data splitting and the same pretrained VAMPnet as a starting point. Afterwards both are trained as described above except for the modified loss function with the same time-lag values as above. The factor in front of the observable loss is always $\lambda=10$ for the results presented here. We rotate the observable used for training and report the results in Fig. 6. Error bars indicate the 70th percentile over 10 training runs. It can be seen that the second timescale is confidentially overestimated by the ordinary model (blue) as intended, which has a direct effect on the folding and unfolding rates. Furthermore, the stationary distribution of the three states is affected, which implies that estimates of expectation val- 
ues cannot be trusted.

Building an observable model (orange) including the expectation of the contact being formed improves the estimation of the stationary distribution overall $(6 \mathrm{~b})$. However, there is no positive effect on the estimation of the kinetics.

Taking both autocorrelations of the contact staying formed and unformed as observables, the observations change (green). It improves the estimates on both stationary and kinetic properties, respectively. Whereas a good performance on the kinetics might be expected, the reason for the positive impact on the stationary distribution becomes only obvious by studying the properties of the autocorrelations. The difference between the two unnormalized autocorrelations is given by the expectation value of the contact (Appendix VIIB). Therefore, if both autocorrelations are matched, the expectation value should be matched, which has a positive effect on the state probabilities as seen above.

Motivated by these findings we tried to match the second implied timescale and the expectation value along training (red). Here, all observables are matched the best. The results suggest, that as few as two experimental observables are sufficient to counteract the bias apparent in this data.

\section{CONCLUSION}

Here we extend the previously proposed reversible deep MSMs 81 by adding features well established for traditional MSMs: the coarse-graining of Markov states to a fewer-state MSM, and the inclusion of experimental restrains into the MSM estimation process. We apply these methods to the study various aspects of the Villin headpiece miniprotein kinetics. We exploit the fact that revDMSMs are faithful probability models and apply transition path theory to study mean first passage times of the folding and unfolding event, where our result coincides with the previously published results 35 . Furthermore, we established an approach how experimental data can be incorporated into the model estimation and how it can possibly compensate for biases in the underlying force fields. The results suggest that it is already valuable to supply few stationary and kinetic information to the model estimation. In addition, the coarse graining method proved valuable in constructing hierarchical models, which give rise to easily interpretable states and allow to study the system on different levels of detail. Finally, we demonstrated how an attention mechanism can draw the attention to residues being important for the classification of the dynamics. Thereby, it could be a valuable tool for practitioners to find targets for mutations to be studied.

Despite these benefits, it remains an open challenge to develop specialized network architectures for protein dynamics analysis, especially the attention network could profit from an architecture where parameters are shared among residues. Furthermore, the inclusion of real experimental observables remains a task for future studies, where the method would need to prove its capabilities to counteract biases of the simulation due to the underlying force field.

\section{ACKNOWLEDGEMENTS}

This work was funded by the European Research Commission (ERC CoG "ScaleCell"), Deutsche Forschungsgemeinschaft (CRC 1114/A04, CRC 958/A04), the Berlin mathematics research center $\mathrm{MATH}+$ (Projects AA1-6 and EF1-2), and the German ministry for research and education (BIFOLD).

\section{DATA AVAILABILITY}

The data that support the findings of this study are available from Lindorff-Larsen et al. ${ }^{[35}$. Restrictions apply to the availability of these data, which were used under license for this study. Data are available from the authors upon reasonable request and with the permission of Lindorff-Larsen et al. $\stackrel{35}{\text {. }}$

\section{REFERENCES}

${ }^{1}$ M. Shirts and V. S. Pande. Screen savers of the world unite! Science, 290:1903-1904, 2000.

${ }^{2}$ J. C. Phillips, R. Braun, W. Wang, J. Gumbart, E. Tajkhorshid, E. Villa, C. Chipot, R. D. Skeel, L. Kalé, and K. Schulten. Scalable molecular dynamics with NAMD. J. Comput. Chem., 26:1781-1802, 2005.

${ }^{3}$ M. Harvey, G. Giupponi, and G. De Fabritiis. Acemd: Accelerated molecular dynamics simulations in the microseconds timescale. J. Chem. Theory Comput., 5:1632-1639, 2009.

${ }^{4}$ I. Buch, M. J. Harvey, T. Giorgino, D. P. Anderson, and G. De Fabritiis. High-throughput all-atom molecular dynamics simulations using distributed computing. J. Chem. Inf. Model., 50:397403, 2010.

${ }^{5}$ D. E. Shaw, P. Maragakis, K. Lindorff-Larsen, S. Piana, R.O. Dror, M.P. Eastwood, J.A. Bank, J.M. Jumper, J.K. Salmon, Y. Shan, and W. Wriggers. Atomic-Level Characterization of the Structural Dynamics of Proteins. Science, 330:341-346, 2010.

${ }^{6}$ P. Eastman, M. S. Friedrichs, J. D. Chodera, R. J. Radmer, C. M. Bruns, J. P. Ku, K. A. Beauchamp, T. J. Lane, L.-P. Wang, D. Shukla, T. Tye, M. Houston, T. Stich, C. Klein, M. R. Shirts, and V. S. Pande. Openmm 4: A reusable, extensible, hardware independent library for high performance molecular simulation. J. Chem. Theory Comput., 9:461-469, 2013.

${ }^{7}$ R. Salomon-Ferrer, A. W. Goetz, D. Poole, S. Le Grand, and R. C. Walker. Routine microsecond molecular dynamics simulations with amber - part ii: Particle mesh ewald. J. Chem. Theory Comput. 9, 9:3878-3888, 2013.

${ }^{8}$ S. Pronk, S. Páll, R. Schulz, P. Larsson, P. Bjelkmar, R. Apostolov, M. R. Shirts, J. C. Smith, P. M. Kasson, D. van der Spoel, B. Hess, and E. Lindahl. Gromacs 4.5: a high-throughput and highly parallel open source molecular simulation toolkit. Bioinformatics, 29:845-854, 2013.

${ }^{9}$ S. Doerr, M. J. Harvey, F. Noé, and G. De Fabritiis. HTMD: High-Throughput Molecular Dynamics for Molecular Discovery. J. Chem. Theory Comput., 12:1845-1852, 2016. 
${ }^{10}$ Ch. Schütte, A. Fischer, W. Huisinga, and P. Deuflhard. A direct approach to conformational dynamics based on Hybrid Monte Carlo. J, Comput. Phys., 151:146-168, 1999.

${ }^{11}$ William C. Swope, Jed W. Pitera, and Frank Suits. Describing protein folding kinetics by molecular dynamics simulations: 1 . Theory. J. Phys. Chem. B, 108:6571-6581, 2004.

${ }^{12}$ F. Noé, I. Horenko, C. Schütte, and J. C. Smith. Hierarchical analysis of conformational dynamics in biomolecules: transition networks of metastable states. J Chem Phys, 126(15), April 2007.

${ }^{13}$ J. D. Chodera, K. A. Dill, N. Singhal, V. S. Pande, W. C. Swope, and J. W. Pitera. Automatic discovery of metastable states for the construction of Markov models of macromolecular conformational dynamics. J. Chem. Phys., 126:155101, 2007.

${ }^{14}$ G. R. Bowman, K. A. Beauchamp, G. Boxer, and V. S. Pande. Progress and challenges in the automated construction of Markov state models for full protein systems. J. Chem. Phys., 131:124101, 2009.

${ }^{15}$ J.-H. Prinz, B. G. Keller, and F. Noé. Probing molecular kinetics with markov models: Metastable states, transition pathways and spectroscopic observables. Phys. Chem. Chem. Phys., 13:1691216927, 2011.

${ }^{16}$ C. Schütte, F. Noé, J. Lu, M. Sarich, and E. Vanden-Eijnden. Markov state models based on milestoning. J. Chem. Phys., 134:204105, 2011.

${ }^{17}$ G. R. Bowman, D. L. Ensign, and V. S. Pande. Enhanced Modeling via Network Theory: Adaptive Sampling of Markov State Models. J. Chem. Theory Comput., 6(3):787-794, 2010.

${ }^{18}$ V. S. Pande, K. A. Beauchamp, and G. R. Bowman. Everything you wanted to know about Markov State Models but were afraid to ask. Methods (San Diego, Calif.), 52(1):99-105, 2010.

${ }^{19}$ M. Weber, K. Fackeldey, and C. Schütte. Set-free markov state model building. J. Chem. Phys., 146:124133,, 2017.

${ }^{20}$ A. L. Ferguson, A. Z. Panagiotopoulos, I. G. Kevrekidis, and P. G. Debenedetti. Nonlinear dimensionality reduction in molecular simulation: The diffusion map approach. Chemical Physics Letters, 509(1-3):1-11, 2011.

${ }^{21}$ A. M. Razavi, W. M. Wuest, and V. A. Voelz. Computational screening and selection of cyclic peptide hairpin mimetics by molecular simulation and kinetic network models. Journal of chemical information and modeling, 54(5):1425-1432, 2014.

${ }^{22}$ D. S. Chekmarev, T. Ishida, and R. M. Levy. Long-time conformational transitions of alanine dipeptide in aqueous solution: Continuous and discrete-state kinetic models. J. Phys. Chem. B, 108:19487-19495, 2004.

${ }^{23}$ S. Sriraman, I. G. Kevrekidis, and G. Hummer. Coarse Master Equation from Bayesian Analysis of Replica Molecular Dynamics Simulations. J. Phys. Chem. B, 109:6479-6484, 2005.

${ }^{24}$ Nicolae-Viorel Buchete and Gerhard Hummer. Coarse master equations for peptide folding dynamics. J. Phys. Chem. B, 112:6057-6069, 2008.

${ }^{25}$ F. Noé, H. Wu, J.-H. Prinz, and N. Plattner. Projected and hidden markov models for calculating kinetics and metastable states of complex molecules. J. Chem. Phys., 139:184114, 2013.

${ }^{26} \mathrm{H}$. Wu and F. Noé. Optimal estimation of free energies and stationary densities from multiple biased simulations. Multiscale Model. Simul., 12:25-54, 2014.

${ }^{27}$ E. Rosta and G. Hummer. Free energies from dynamic weighted histogram analysis using unbiased markov state model. J. Chem. Theory Comput., 11:276-285, 2015.

${ }^{28} \mathrm{H}$. Wu and F. Noé. Gaussian markov transition models of molecular kinetics. J. Chem. Phys., 142:084104, 2015.

${ }^{29} \mathrm{H}$. Wu, F. Paul, C. Wehmeyer, and F. Noé. Multiensemble markov models of molecular thermodynamics and kinetics. Proc. Natl. Acad. Sci. USA, 113(23):E3221-E3230, 2016.

${ }^{30} \mathrm{~W}$. E and E. Vanden-Eijnden. Metastability, conformation $d y-$ namics, and transition pathways in complex systems., pages 3865. Multiscale Modelling and Simulation. Springer, 2004.

${ }^{31}$ P. Tiwary and M. Parrinello. From metadynamics to dynamics. Phys. Rev. Lett., 111:230602, 2013.
${ }^{32}$ J. M. L. Ribeiro, P. Bravo, Y. W., and P. Tiwary. Reweighted autoencoded variational bayes for enhanced sampling (rave). $J$. Chem. Phys., 149:072301, 2018.

${ }^{33}$ W. Chen and A. L. Ferguson. Molecular enhanced sampling with autoencoders: On-the-fly collective variable discovery and accelerated free energy landscape exploration. Journal of computational chemistry, 39(25):2079-2102, 2018.

${ }^{34}$ F. Noé, C. Schütte, E. Vanden-Eijnden, L. Reich, and T. R. Weikl. Constructing the full ensemble of folding pathways from short off-equilibrium simulations. accepted for Proc. Natl. Acad. Sci. USA on September 16 2009, 2009.

${ }^{35}$ K. Lindorff-Larsen, S. Piana, R. O. Dror, and D. E. Shaw. How fast-folding proteins fold. Science, 334:517-520, 2011.

${ }^{36}$ I. Buch, T. Giorgino, and G. De Fabritiis. Complete reconstruction of an enzyme-inhibitor binding process by molecular dynamics simulations. Proc. Natl. Acad. Sci. USA, 108:10184-10189, 2011.

${ }^{37}$ D.-A. Silva, G. R. Bowman, A. Sosa-Peinado, and X. Huang. A role for both conformational selection and induced fit in ligand binding by the lao protein. PLoS Comput. Biol., 7:e1002054, 2011.

${ }^{38}$ N. Plattner and F. Noé. Protein conformational plasticity and complex ligand binding kinetics explored by atomistic simulations and markov models. Nat. Commun., 6:7653, 2015.

${ }^{39}$ P. Tiwary, V. Limongelli, M. Salvalaglio, and M. Parrinello. Kinetics of protein-ligand unbinding: Predicting pathways, rates, and rate-limiting steps. Proc. Natl. Acad. Sci. USA, 112:E386E391, 2014

${ }^{40}$ N. Plattner, S. Doerr, G. De Fabritiis, and F. Noé. Proteinprotein association and binding mechanism resolved in atomic detail. Nat. Chem., 9:1005-1011, 2017.

${ }^{41}$ M. Sarich, F. Noé, and C. Schütte. On the approximation quality of markov state models. Multiscale Model. Simul., 8:1154-1177, 2010.

${ }^{42}$ F. Noé, S. Doose, I. Daidone, M. Löllmann, J. D. Chodera, M. Sauer, and J. C. Smith. Dynamical fingerprints for probing individual relaxation processes in biomolecular dynamics with simulations and kinetic experiments. Proc. Natl. Acad. Sci. USA, 108:4822-4827, 2011.

${ }^{43}$ B. G. Keller, J.-H. Prinz, and F. Noé. Markov models and dynamical fingerprints: Unraveling the complexity of molecular kinetics. Chem. Phys., 396:92-107, 2012.

${ }^{44}$ Wei Zhuang, Raymond Z. Cui, Daniel-Adriano Silva, and Xuhui Huang. Simulating the T-Jump-Triggered Unfolding Dynamics of trpzip2 Peptide and Its Time-Resolved IR and Two-Dimensional IR Signals Using the Markov State Model Approach. J. Phys. Chem. B, 115:5415-5424, 2011.

${ }^{45}$ B. Lindner, Z. Yi, J.-H. Prinz, J. C. Smith, and F. Noé. Dynamic Neutron Scattering from Conformational Dynamics I: Theory and Markov models. J. Chem. Phys., 139:175101, 2013.

${ }^{46}$ J. D. Chodera and F. Noé. Probability distributions of molecular observables computed from markov models. ii: Uncertainties in observables and their time-evolution. J. Chem. Phys., 133:105102, 2010.

${ }^{47}$ V. A. Voelz, V. R. Singh, W. J. Wedemeyer, L. J. Lapidus, and V. S. Pande. Unfolded-state dynamics and structure of protein 1 characterized by simulation and experiment. Journal of the American Chemical Society, 132(13):4702-4709, 2010.

${ }^{48}$ G. Zhou, G. A. Pantelopulos, S. Mukherjee, and V. A. Voelz. Bridging microscopic and macroscopic mechanisms of p53-mdm2 binding with kinetic network models. Biophysical journal, 113(4):785-793, 2017.

${ }^{49}$ C. R. Schwantes and V. S. Pande. Improvements in markov state model construction reveal many non-native interactions in the folding of nt19. J. Chem. Theory Comput., 9:2000-2009, 2013.

${ }^{50}$ G. Perez-Hernandez, F. Paul, T. Giorgino, G. D Fabritiis, and Frank Noé. Identification of slow molecular order parameters for markov model construction. J. Chem. Phys., 139:015102, 2013.

${ }^{51}$ F. Noé and F. Nüske. A variational approach to modeling slow processes in stochastic dynamical systems. Multiscale Model. 
Simul., 11:635-655, 2013.

${ }^{52}$ F. Nüske, B. G. Keller, G. Pérez-Hernández, A. S. J. S. Mey, and F. Noé. Variational approach to molecular kinetics. J. Chem. Theory Comput., 10:1739-1752, 2014.

${ }^{53} \mathrm{H}$. Wu and $\mathrm{F}$ Noé. Variational approach for learning markov processes from time series data. Journal of Nonlinear Science, Aug 2019.

${ }^{54}$ A. Mardt, L. Pasquali, H. Wu, and F. Noé. Vampnets: Deep learning of molecular kinetics. Nat. Commun., 9:5, 2018.

${ }^{55}$ W. Chen, H. Sidky, and A. L. Ferguson. Nonlinear discovery of slow molecular modes using state-free reversible vampnets. The Journal of Chemical Physics, 150(21):214114, Jun 2019.

${ }^{56}$ T. Xie, A. France-Lanord, Y. Wang, Y. Shao-Horn, and J. C. Grossman. Graph dynamical networks for unsupervised learning of atomic scale dynamics in materials. Nature communications, 10(1):2667, 2019

${ }^{57} \mathrm{~W}$. E and E. Vanden-Eijnden. Towards a Theory of Transition Paths. J. Stat. Phys., 123:503-523, 2006.

${ }^{58}$ P. Metzner, C. Schütte, and E. Vanden-Eijnden. Transition Path Theory for Markov Jump Processes. Multiscale Model. Simul., 7:1192-1219, 2009.

${ }^{59}$ F. Noé, C. Schütte, E. Vanden-Eijnden, L. Reich, and T. R. Weikl. Constructing the full ensemble of folding pathways from short off-equilibrium simulations. Proc. Natl. Acad. Sci. USA, 106:19011-19016, 2009.

${ }^{60}$ T. Löhr, K. Kohlhoff, G. T Heller, C. Camilloni, and M. Vendruscolo. A kinetic ensemble of the alzheimer's A $\beta$ peptide. Nature Computational Science, 1(1):71-78, 2021.

${ }^{61}$ P. Deuflhard, W. Huisinga, A. Fischer, and C. Schütte. Identification of almost invariant aggregates in reversibly nearly uncoupled Markov chains. Lin. Alg. Appl., 315:39-59, 2000.

${ }^{62} \mathrm{P}$. Deuflhard and M. Weber. Robust perron cluster analysis in conformation dynamics. In M. Dellnitz, S. Kirkland, M. Neumann, and C. Schütte, editors, Linear Algebra Appl., volume 398C, pages 161-184. Elsevier, New York, 2005.

${ }^{63} \mathrm{~K}$. Fackeldey and M. Weber. Genpcca - markov state models for non-equilibrium steady states. WIAS Report, 29:70-80, 2017.

${ }^{64}$ S. Olsson, H. Wu, F. Paul, C. Clementi, and F. Noé. Combining experimental and simulation data of molecular processes via augmented markov models. Proc. Natl. Acad. Sci. USA, 114:82658270, 2017.

${ }^{65}$ G. Hummer and J. Köfinger. Bayesian ensemble refinement by replica simulations and reweighting. J. Chem. Phys., 143:243150, 2015.

${ }^{66} \mathrm{~J}$. W. Pitera and J. D. Chodera. On the use of experimental observations to bias simulated ensembles. Journal of chemical theory and computation, 8(10):3445-3451, 2012.

${ }^{67}$ W. Boomsma, J. Ferkinghoff-Borg, and K. Lindorff-Larsen. Combining experiments and simulations using the maximum entropy principle. PLoS Comput Biol, 10(2):e1003406, 2014.

${ }^{68}$ K. A. Beauchamp, V. S. Pande, and R. Das. Bayesian energy landscape tilting: towards concordant models of molecular ensembles. Biophysical journal, 106(6):1381-1390, 2014.

${ }^{69}$ A. Cavalli, C. Camilloni, and M. Vendruscolo. Molecular dynamics simulations with replica-averaged structural restraints generate structural ensembles according to the maximum entropy principle. The Journal of chemical physics, 138(9):03B603, 2013.

${ }^{70}$ H. T. A. Leung, O. Bignucolo, R. Aregger, S. A. Dames, A. Mazur, S. Bernéche, and S. Grzesiek. A rigorous and efficient method to reweight very large conformational ensembles using average experimental data and to determine their relative information content. Journal of chemical theory and computation, 12(1):383-394, 2016.

${ }^{71}$ G. Montavon, W. Samek, and K.-R. Müller. Methods for interpreting and understanding deep neural networks. Digital Signal Processing, 73:1-15, 2018.

${ }^{72}$ M. T. Ribeiro, S. Singh, and C. Guestrin. " why should i trust you?" explaining the predictions of any classifier. In Proceedings of the 22nd ACM SIGKDD international conference on knowledge discovery and data mining, pages 1135-1144, 2016.
${ }^{73}$ S. M. Lundberg and S.-I. Lee. A unified approach to interpreting model predictions. In Advances in neural information processing systems, pages 4765-4774, 2017.

${ }^{74} \mathrm{R}$. C. Fong and A. Vedaldi. Interpretable explanations of black boxes by meaningful perturbation. In Proceedings of the IEEE International Conference on Computer Vision, pages 3429-3437, 2017.

${ }^{75}$ V. Mnih, N. Heess, A. Graves, et al. Recurrent models of visual attention. In Advances in neural information processing systems, pages 2204-2212, 2014.

${ }^{76}$ K. Xu, J. Ba, R. Kiros, K. Cho, A. Courville, R. Salakhudinov, R. Zemel, and Y. Bengio. Show, attend and tell: Neural image caption generation with visual attention. In International conference on machine learning, pages 2048-2057, 2015.

${ }^{77}$ D. Bau, B. Zhou, A. Khosla, A. Oliva, and A. Torralba. Network dissection: Quantifying interpretability of deep visual representations. In Proceedings of the IEEE conference on computer vision and pattern recognition, pages 6541-6549, 2017.

${ }^{78}$ P.-J. Kindermans, K. T. Schütt, M. Alber, K.-R. Müller, D. Erhan, B. Kim, and S. Dähne. Learning how to explain neural networks: Patternnet and patternattribution. arXiv preprint arXiv:1705.05598, 2017.

${ }^{79}$ R. R. Selvaraju, M. Cogswell, A. Das, R. Vedantam, D. Parikh, and D. Batra. Grad-cam: Visual explanations from deep networks via gradient-based localization. In Proceedings of the IEEE international conference on computer vision, pages 618626, 2017

${ }^{80}$ Q. Zhang, Y. Yang, H. Ma, and Y. N. Wu. Interpreting cnns via decision trees. In Proceedings of the IEEE Conference on Computer Vision and Pattern Recognition, pages 6261-6270, 2019.

${ }^{81}$ A. Mardt, L. Pasquali, F. Noé, and H. Wu. Deep learning markov and koopman models with physical constraints. In Mathematical and Scientific Machine Learning, pages 451-475. PMLR, 2020.

${ }^{82} \mathrm{~S}$. Röblitz and M. Weber. Fuzzy spectral clustering by PCCA+: application to Markov state models and data classification. Adv. Data Anal. Classif., 7:147-179, 2013.

${ }^{83}$ A. Paszke, S. Gross, F. Massa, A. Lerer, J. Bradbury, G. Chanan, T. Killeen, Z. Lin, N. Gimelshein, L. Antiga, et al. Pytorch: An imperative style, high-performance deep learning library. In Advances in neural information processing systems, pages 80268037, 2019.

${ }^{84}$ D. P. Kingma and J. Ba. Adam: A method for stochastic optimization. CoRR, abs/1412.6980, 2014

${ }^{85} \mathrm{~B}$. E. Husic and F. Noé. Deflation reveals dynamical structure in nondominant reaction coordinates. The Journal of Chemical Physics, 151(5):054103, 2019.

${ }^{86}$ M. K. Scherer, B. Trendelkamp-Schroer, F. Paul, G. PerezHernandez, M. Hoffmann, N. Plattner, C. Wehmeyer, J.-H. Prinz, and F. Noé. PyEMMA 2: A software package for estimation, validation and analysis of Markov models. J. Chem. Theory Comput., 11:5525-5542, 2015.

\section{APPENDIX}

\section{A. Interpretability via attention mechanism}

In applications it is of great interest to understand how the network defines states. For proteins this translates to understand which residues are important for the classification. Here, we focus on an attention formalism using an additional network $\mathbf{A}\left(\mathbf{x}_{t}\right)$, which takes a configuration $\mathbf{x}_{t}$ as an input. The input of the classification network transforms to $\chi\left(\mathbf{A}\left(\mathbf{x}_{t}\right) \odot \mathbf{x}_{t}\right)$, where the configuration is scaled by the element wise multiplication with the output of the attention network ${ }^{75576}$. Due to a softmax output 


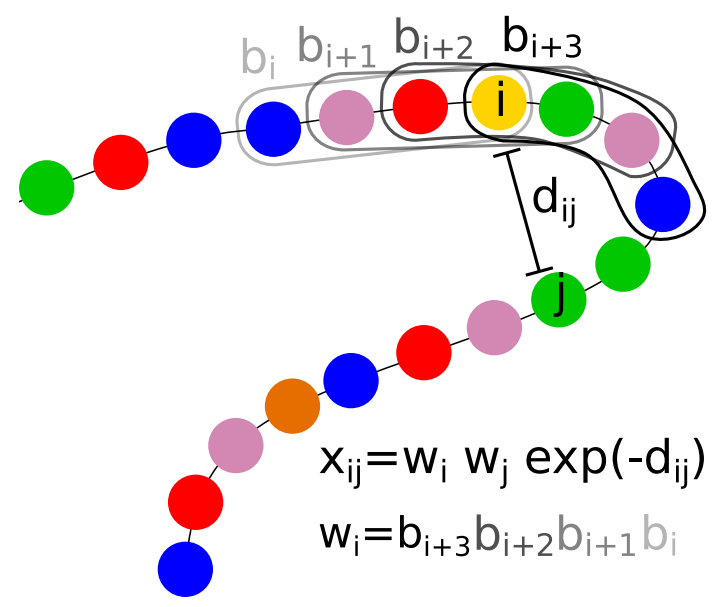

Figure 7. Schematic of the attention mechanism, how to assign attention weights for the input distances given the output values of the attention network $\mathbf{b}(t)=\mathbf{A}(\mathbf{x}(t))$. The weight $w_{i}$ of residue $i$ is constituted of all window size $B=4$ many values $w_{i}=\prod_{l=0}^{B-1} b_{i+l}$. A weight $b_{i}$ is shared among all neighbored residues within the window $i$ along the chain. Thereby, primary structural information is fed into the network. Since a softmax output function for the network $\mathbf{A}$ is chosen, the values b can be interpreted as importance values for the classification. By applying a softmax function to the weights $\mathbf{w}$ the same interpretation holds for the residue weights. Since we pass $x_{i j}=\exp \left(-d_{i j}\right)$ into $\chi$, we scale each input distance $d_{i j}$ with the corresponding weights for the residues $i, j: x_{i j}=w_{i} w_{j} \exp \left(-d_{i j}\right)$.

function the attention network indicates how important each dimension of the input is.

Since for proteins it is preferable to use internal coordinates as inputs such as distances between residues $d_{i j}$, but scores for each residue are easier interpretable, we define $\mathbf{A}$ to predict weights for each residue $w_{i}$. Thereby, the input to $\chi$ will be scaled as $x_{i j}=w_{i} w_{j} f\left(d_{i j}\right)$, where $f$ can be an arbitrary function. Additionally, we add primary information of the chain of residues by estimating the weights $w_{i}$ via values $\mathbf{b}=\mathbf{A}\left(\mathbf{x}_{t}\right)$, which are shared within a window along the chain (Fig. 7). With the window size $B$, a weight of a residue $w_{i}$ is given by:

$$
w_{i}=\prod_{j=0}^{B-1} b_{i+j},
$$

where the attention network $\mathbf{A}$ outputs a vector of dimension $\operatorname{dim}(\mathbf{b})=N+B-1$ assigning with a softmax function importance values to each window. It can be interpreted as a sliding window passing over the $N$ residue chain, which will result into a smoother attention representation along the chain. Afterwards, the softmax function can be applied over the weights of the residues:

$$
\bar{w}_{i}=\frac{\exp \left(w_{i}\right)}{\sum_{j} \exp w_{j}} .
$$

In the case of large proteins, where a lower resolution of attention weights might be favorable, an additional skip value can be introduced defining how many residues are skipped before a new window is defined. The weights of the attention network are updated with a smaller learning rate during the pretraining of the VAMPnet, since the gradients acting from the VAMPnet $\chi$ could be misleading due to an unfavorable starting configuration. We are aware that the architectures of neural network are fast developing, nonetheless we think that the sliding window formulation is of general benefit due to its flexibility.

\section{B. Matching two dependent time correlations}

Given a microscopic observable $a_{1}$ and defining $a_{2}=$ $1-a_{1}$, the expectation value of $a_{2}$ is:

$$
\mathbb{E}\left[a_{2}\right]=\mathbb{E}\left[1-a_{1}\right]=1-\mathbb{E}\left[a_{1}\right] .
$$

Therefore, it is of no use to match both expectation values $\mathbb{E}\left[a_{1}\right], \mathbb{E}\left[a_{2}\right]$. However, if we inspect the time correlation:

$$
\begin{aligned}
\mathbb{E}\left[a_{2}(t) a_{2}(t+\tau)\right] & =\mathbb{E}\left[\left(1-a_{1}(t)\right)\left(1-a_{1}(t+\tau)\right)\right] \quad(25) \\
& =1-\mathbb{E}\left[a_{1}(t)\right]-\mathbb{E}\left[a_{1}(t+\tau)\right]+\mathbb{E}\left[a_{1}(t) a_{1}(t+\tau)\right]
\end{aligned}
$$

it is obvious that matching both time correlations is equivalent to matching one time correlation and the corresponding expectation value.

\section{Connection between timescales and folding/unfolding rates}

Given experimental rates (time interval per event) for folding $r_{\text {on }}$ and unfolding $r_{\text {off }}$ it can be seen as a two state (folded and unfolded) Markov process with a transition matrix $\mathbf{P}$. The probabilities of jumping between these states within a time interval $\tau$ can be calculated as the inverse rates $k_{\mathrm{on}}=\frac{1}{r_{\mathrm{on}}} \tau$. The transition matrix reads then:

$$
\mathbf{P}=\left[\begin{array}{cc}
1-k_{\mathrm{on}} & k_{\mathrm{on}} \\
k_{\mathrm{off}} & 1-k_{\mathrm{off}}
\end{array}\right]
$$


The eigenvalues of $\mathbf{P}$ are $[1, \lambda]$, where $\lambda$ is the eigenvalue corresponding to the folding process. Via the trace the connection is recognizable:

$$
\begin{aligned}
\operatorname{tr}(\mathbf{P}) & =1+\lambda=1-k_{\mathrm{on}}+1-k_{\mathrm{off}} \\
\Rightarrow \lambda & =1-k_{\mathrm{on}}-k_{\mathrm{off}}=1-\tau\left(\frac{1}{r_{\mathrm{on}}}+\frac{1}{r_{\mathrm{off}}}\right) .
\end{aligned}
$$

\section{Pretraining the VAMPnet}

In the original paper a pretraining of the VAMPnet with a symmetrized VAMP-loss is recommended in or- der to achieve a more crisp assignment of the classification network. Since there is no clear motivation about this particular procedure, we modified the pretraining by adding to the VAMP-2 score the term $\operatorname{tr}\left(\mathbf{C}_{00}\right)$ which will maximize the eigenvalues of the matrix and therefore favors harder state assignments. The updated loss changes to the following:

$$
L=-\mathrm{VAMP}-2-\lambda \operatorname{tr}\left(\mathbf{C}_{00}\right),
$$

where $\lambda$ balances the two terms and can be set to zero during the following unperturbed training phase. Thereby, we give a clear motivation that the additional term pushes the network into a more favorable region during training. 